\title{
Novelty Search for Soft Robotic Space Exploration
}

\author{
Georgios Methenitis ${ }^{*}$ \\ Centrum Wiskunde \& \\ Informatica \\ (CWI) \\ Amsterdam, The Netherlands \\ Georgios.Methenitis@cwi.nl
}

\author{
Daniel Hennes \\ Advanced Concepts Team \\ European Space Agency \\ (ESTEC) \\ Noordwijk, The Netherlands \\ Daniel.Hennes@esa.int
}

Arnoud Visser

University of Amsterdam

Amsterdam, The Netherlands

A.Visser@uva.nl

\author{
Dario Izzo \\ Advanced Concepts Team \\ European Space Agency \\ (ESTEC) \\ Noordwijk, The Netherlands \\ Dario.lzzo@esa.int
}

\begin{abstract}
The use of soft robots in future space exploration is still a far-fetched idea, but an attractive one. Soft robots are inherently compliant mechanisms that are well suited for locomotion on rough terrain as often faced in extra-planetary environments. Depending on the particular application and requirements, the best shape (or body morphology) and locomotion strategy for such robots will vary substantially. Recent developments in soft robotics and evolutionary optimization showed the possibility to simultaneously evolve the morphology and locomotion strategy in simulated trials. The use of techniques such as generative encoding and neural evolution were key to these findings. In this paper, we improve further on this methodology by introducing the use of a novelty measure during the evolution process. We compare fitness search and novelty search in different gravity levels and we consistently find novelty-based search to perform as good as or better than a fitness-based search, while also delivering a greater variety of designs. We propose a combination of the two techniques using fitness-elitism in novelty search to obtain a further improvement. We then use our methodology to evolve the gait and morphology of soft robots at different gravity levels, finding a taxonomy of possible locomotion strategies that are analyzed in the context of space-exploration.
\end{abstract}

\section{CCS Concepts}

-Computing methodologies $\rightarrow$ Artificial intelligence; Evolutionary robotics; Search methodologies; Control methods;

\footnotetext{
*Based on the master's thesis [1], work conducted under the supervision of both the University of Amsterdam and the Advanced Concepts Team (ESTEC).

Permission to make digital or hard copies of all or part of this work for personal or classroom use is granted without fee provided that copies are not made or distributed for profit or commercial advantage and that copies bear this notice and the full citation on the first page. Copyrights for components of this work owned by others than ACM must be honored. Abstracting with credit is permitted. To copy otherwise, or republish, to post on servers or to redistribute to lists, requires prior specific permission and/or a fee. Request permissions from permissions@acm.org.

GECCO '15, July 11 - 15, 2015, Madrid, Spain

(c) 2015 ACM. ISBN 978-1-4503-3472-3/15/07 . \$ $\$ 15.00$

DOI: http://dx.doi.org/10.1145/2739480.2754731
}

\section{Keywords}

Soft Robotics; Locomotion; Novelty Search; CPPN; CPPNNEAT; VoxCAD; Space Exploration

\section{INTRODUCTION}

Robotic exploration of extraterrestrial bodies is a challenging endeavour that requires the deployment of advanced technological solutions. The Huygens descent on Titan, the landing of Philae on the comet $67 \mathrm{P} /$ Churyumov-Gerasimenko or the daily accounts of the three martian rovers Spirit, Opportunity and Curiosity are incredible achievements that were made possible by our ever increasing technological capabilities. One challenge, in all of these missions, is that of mobility. Advanced mobility solutions for exoplanetary rovers are the subject of active research. It is not uncommon in this research field to find ideas drawing inspiration from biology. For example, this was the case for the Mars tumbleweed exploration concept $[2,3]$, the lunar ALI exploration concept [4], or the many legged robots, inspired by insect locomotion, that are being studied in the context of exoplanetary exploration. The locomotion strategy and the body morphology of these robots, though closely related, were not co-designed and were treated as rather uncoupled entities. In this work, we are interested in investigating the possibility to simultaneously design the morphology and the gait of robots as to adapt their locomotion to different extraterrestrial environments defined by different terrain types and gravity strengths. The effects of gravity, slopes, and stiffness on the gaits evolved by a quadruped robot in dynamic walking and running was researched in $[5,6]$. Hildebrand gait diagrams [7] were employed to analyze gaits and prove their stability under varying planetary conditions. The robot morphology, though, was kept fixed and only the leg lengths and the controller were subject to optimization. The simultaneous optimization of gaits and morphology, using evolutionary techniques is, though, possible. In the work of Sims [8] a generative (or indirect) encoding was used to enable the evolution of virtual creatures adapted for jumping, walking or swimming. The idea has been a source of inspiration for a large number of later works and recently, the practical possibility to evolve soft material robots [9] able to produce locomotion has been proved. The evolution of soft structures in a virtual environment was later also studied 

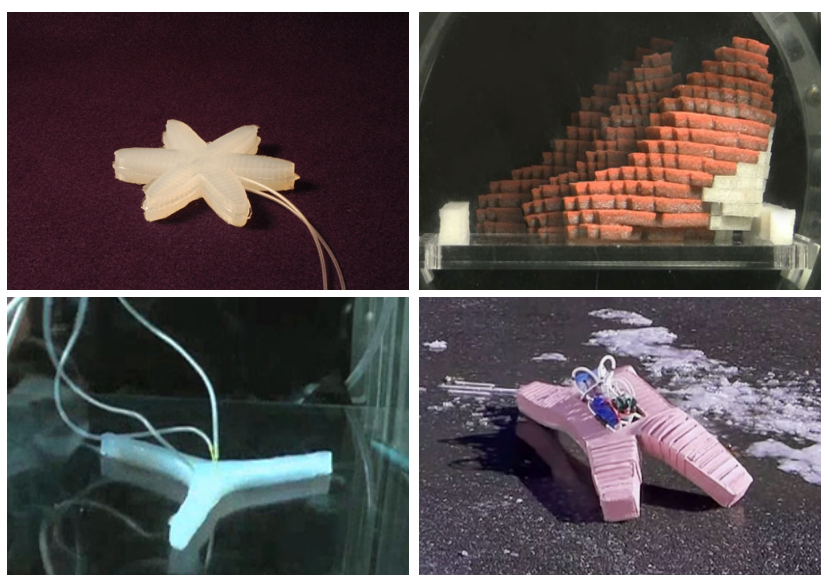

Figure 1: Soft robots can be actuated through air pressure tubes (top-left), pressure variations (topright), or internal explosions (bottom-left). Autonomously actuated soft robot [20], it is able to withstand extreme temperatures and variant terrain types (bottom-right).

in [10] where a powerful generative encoding, CPPNs [11], was used to generate soft voxel-formed three dimensional structures, coupled with the use of NEAT algorithm which ensures the increasing complexity of the networks produced. The interest of soft robotics $[12,13]$ for space exploration has been recently well defined by Lin, Leisk and Trimmer [14] and summarized in three points: a) intrinsically safe (rather than "control safe") b) morph-able (size-changing and versatile) and c) gravity independent (able to move in any orientation). Having no rigid parts in their design (See Fig. 1), the degrees of freedom of a soft robot are infinite and the possible ways of motion can become extremely rich and hard to control. In this paper we build upon previous work [10] first improving significantly the methodology by considering the evolutionary process as driven by novelty $[15,16,17$, 18] rather than by fitness. A similar idea was investigated in [19] in the context of rigid robots and proved to result in a far more exploratory algorithm in the space of morphologies. We then obtain a further performance boost combining novelty search with fitness-elitism and we use the resulting algorithm to evolve a number of robots and locomotion patterns adapted to different planetary conditions. Given the extreme complexity of the task and the early research stages of the tools used, we are not as much interested in the practical use of the designs evolved as in their use to inspire future advanced robotic exploration missions.

\section{BACKGROUND}

We start by introducing VoxCad, the open source simulator used throughout this study to analyze soft robot morphologies and locomotion strategies. Shape and structure of the studied robots are expressed using a generative encoding technique and evolved using a neuro-evolutionary algorithm called CPPN-NEAT. In particular, we follow the approach detailed in previous work [10] and briefly summarize the key aspects of the resulting setup here.

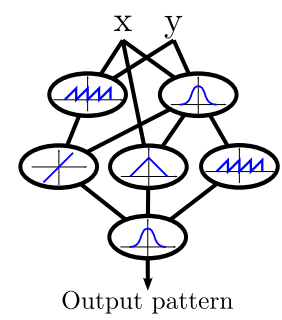

Figure 2: Compositional pattern-producing networks have identical network structure with artificial neural networks while they make use of a canonical set of activation functions.

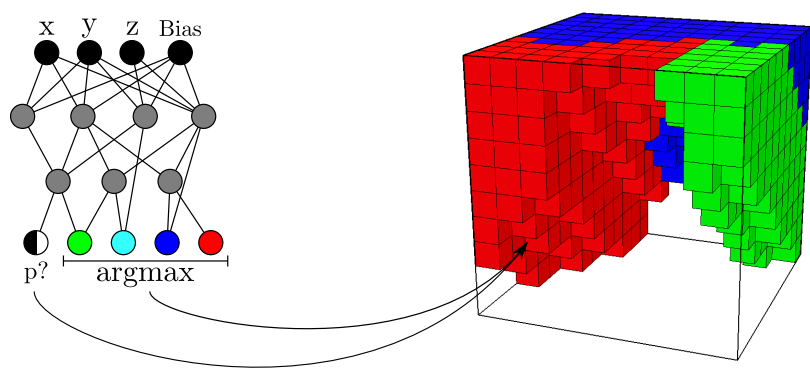

Figure 3: Each genotype (CPPN) is queried for every coordinate inside the lattice, its outputs determine the presence of a voxel and the type of its material.

\subsection{VoxCad simulator}

VoxCad [21] is a simulator targeted to mimic the physics of soft material, while accurately reflecting deformations and interactions. The workspace is represented as a 3D-lattice, where each voxel is of a certain material type. Materials can be actuated (i.e. expansion or contraction) by an external trigger. External actuation is achieved by varying the temperature of the environment and thus exploiting the various material properties. Modifying the material properties results in soft or hard material that either does (active) or does not react (passive) to temperature variations.

\subsection{Generative encoding}

We use the technique of Compositional Pattern-Producing Networks (CPPNs) [11] as a generative encoding to produce morphologies (i.e. shape and structure) of simulated soft robots. CPPNs are artificial neural networks with an extended set of activation functions. The set of activation functions can include repetitive, symmetrical, and linear functions; an example is depicted in Figure 2. The CPPNs are queried for every coordinate of the lattice space to form a soft robot shape as well as to define the distribution of the materials. The input nodes (neurons) of the CPPN are assigned to normalized coordinates: ${ }^{1} x, y, z \in[-1,1]$. The output of the network defines if the queried voxel is enabled and which material is used. The generative encoding of voxelyzed soft robots through CPPNs is shown in Figure 3.

\footnotetext{
${ }^{1}$ Therefore, the morphology can be sampled at different lattice resolutions.
} 


\subsection{CPPN-NEAT}

As CPPNs are artificial neural networks, yet with a richer set of activation function, we can deploy neuro-evolutionary algorithms to evolve these networks. Specifically, we use a technique called NeuroEvolution of Augmenting Topologies (NEAT) [22] with minor modifications in order to be applicable to CPPNs, the resulting algorithm is known as CPPNNEAT [11]. During evolution, CPPN-NEAT modifies not only the weights, but also the structure of the network and the activation functions. As such, the number of hidden layers and neurons does not need to be fixed in advance.

\section{METHODOLOGY}

As outlined in the previous section, the use of soft robot simulator (VoxCad) and generative encoding in combination with neuro-evolution through CPPN-NEAT paves the way for successfully evolving complex morphologies for soft robots. However, in previous work [10], the diversity of evolved creatures was limited. Different locomotion profiles were predominantly the result of including secondary costs (e.g., cost for actuated voxels or voxel connections) in the fitness function. We now describe our approach based on novelty search that ensures intrinsic diversity without the need for a "hand-coded" objective that is subjective to the designers domain knowledge and may not generalize to exoplanetary environments with a variety of gravity levels. In addition, we describe how fitness-elitism can be incorporated in novelty search to combine the best of both worlds.

\subsection{Novelty search}

Unlike traditional fitness-based search, novelty search [15, $16,17,18]$ is an alternative way of optimization. Novelty search, evaluates new candidates with respect to their "novelty" when compared to all previously found solutions and not based on an objective function. The "novelty" measure is defined in the behavior space, which is a function of the phenotype. As an example, one can think of the behavior as the trajectory of a robot controlled by a neural network. A "novel" candidate will exhibit a trajectory that differs from all previously recorded trajectories (i.e. in the behavior space) and novelty is not determined by the difference in the weights of the neural network (i.e the genome search space).

For a behavior $x$, a novelty measure $f(x)$ determines how different the specific behavior is in respect to a set of other behaviors $\mathcal{S}$. We follow the definition in [15, 16], where the novelty measure is defined as sparseness measuring the average distance from the $k$-closest behaviors:

$$
f(x)=\frac{1}{k} \sum_{i=1}^{k} \operatorname{dist}\left(x, \mathcal{S}_{i}\right),
$$

where $S$ is a sorted set of the closest behaviors and dist is a distance function between two behaviors.

In the context of soft robots, we define a behavior as the way the robot interacts with the environment during a VoxCad simulation run. Every aspect of the soft robot movement that can be observed, can also be used to describe the behavior. It is expected that behaviors that indirectly contain fitness-related information (i.e. displacement) will be more successful in terms of the original fitness function. Figure 4 presents all behaviors used for novelty computation during our study, in addition to behaviors that relate
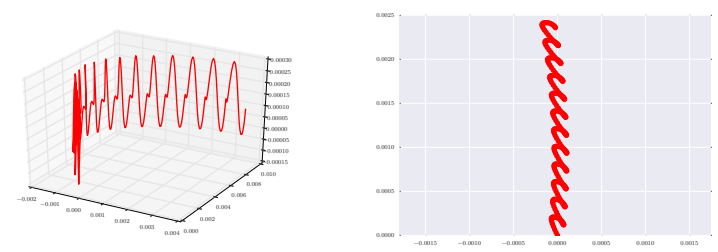

(a) Three and two dimensional trajectories of the soft robots.
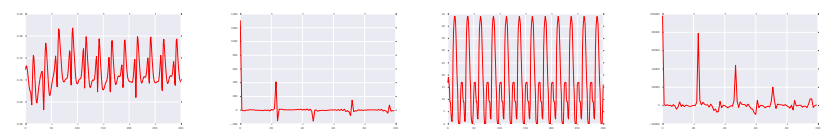

(b) Pace and discrete (c) Voxels touching the Fourier transformation of ground and discrete the same signal. Fourier transformation of the same signal.
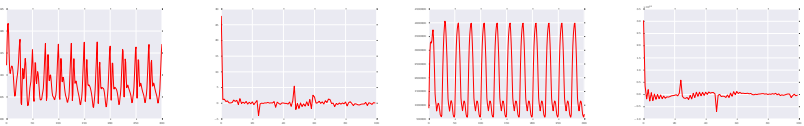

(d) Maximum pressure and (e) Kinetic energy and disdiscrete Fourier transfor- crete Fourier transformamation of the same signal. tion of the same signal.

Figure 4: Observed behaviors of the soft robots used for the sparsity computation in novelty search.

to displacement, e.g., trajectories and pace, we also include others that do not have this obvious relation, e.g., number of voxels touching the ground, kinetic energy, and pressure. For all recorded behavior metrics a constant sampling rate ensures that all signals have the same length. Furthermore, the discrete Fourier transformation of one dimensional signals was used to define other behavior types. The dominant frequencies of the pace, voxels touching the ground, pressure, and kinetic energy are expected to be descriptive of the locomotion strategies the evolved soft robots exhibit.

In order to compute the novelty measure (see Equation (1)) for a trajectory behavior, we need to define the distance function dist. By definition, all trajectories start at the origin and trajectory points are sampled at the same constant rate. Trajectories are rotated so that the average over all trajectory points falls on the $x$-axis, thus resulting in a rotation-invariant measure. Hence, the difference in two behaviors $\mathcal{S}_{i}$ and $\mathcal{S}_{j}$ can be computed as the sum over the point absolute differences between the corresponding trajectories $\boldsymbol{t}_{i}$ and $\boldsymbol{t}_{j}$ :

$$
\begin{gathered}
\mathcal{S}_{i}=\boldsymbol{t}_{i}=t_{i, 1}, t_{i, 2}, \ldots, t_{i, N} \\
\mathcal{S}_{j}=\boldsymbol{t}_{j}=t_{j, 1}, t_{j, 2}, \ldots, t_{j, N} \\
\operatorname{dist}\left(\mathcal{S}_{i}, \mathcal{S}_{j}\right)=\boldsymbol{t}_{i}-\boldsymbol{t}_{j}=\sum_{k=1}^{N}\left\|t_{i, k}-t_{j, k}\right\|
\end{gathered}
$$

where $N$ is the number of sampled coordinate points. In the same way we can define the distance between other types of behaviors and subsequently compute the sparseness (see Equation (1)) a.k.a the novelty measure. For the discrete Fourier transformation of the one dimensional signals only the absolute difference in the first twenty coefficients is measured. 


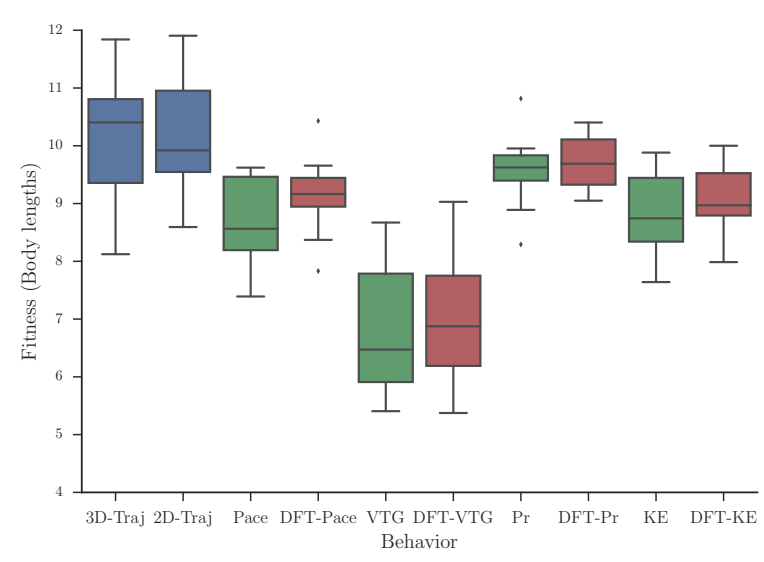

Figure 5: Boxplots of the champion fitness under 10 variant defined behaviors for novelty search. Blue are trajectory type behaviors (3D and 2D), green are 1D signals (pace, voxels touching the ground (VTG), pressure (Pr), and kinetic energy (KE)). Red is the discrete Fourier transformation (DFT$<$ type $>$ ) of the 1D signals.

\subsection{Fitness-elitism in novelty search}

While novelty search promotes diversity by rewarding novel individuals, elitism ensures that the best genetic material is passed on from generation to generation. In particular, we introduce elitism in CPPN-NEAT by carrying-over the best individual of each species so that they can contribute with their beneficial genes later in the evolutionary process. We distinguish between fitness-elitism, where the "best" individuals are selected based on the fitness function and novelty-elitism, where the individuals with the highest sparseness are protected from modification. When combining fitness-elitism with novelty search, the fittest individual is carried over to the next generation and serves in the gene pool to possibly spawn novel yet fit individuals thus combining the best of both worlds, process similar to novelty-based multiobjectivization [23]. Going forward, we deploy novelty search with and without fitness-elitism to study the effectiveness of this approach.

\subsection{Experimental setup}

Each experiment consists of 10 runs with the following fixed settings. The population size is set to 30 individuals and the maximum number of generations per run is set to 1000 . Due to the extremely computationally demanding simulations, not all experiments could be carried out using a VoxCad lattice of $10 \times 10 \times 10$, where applicable deviating dimensions are indicated. We use two externally activated materials with non-zero and opposite thermal expansion coefficients, colored Red and Green. The two additional materials represent non-actuated tissue, representing soft tissue (Cyan voxels) with a five times smaller elastic modulus of their material than hard tissue (Blue voxels). For neuroevoluation we use the HyperNEAT ${ }^{2}$ package; all parameter settings of CPPN-NEAT are the same as detailed in [10].

\footnotetext{
${ }^{2}$ HyperNEAT v4.0 C++ by J. Gauci code (url: https://
} github.com/MisterTea/HyperNEAT)

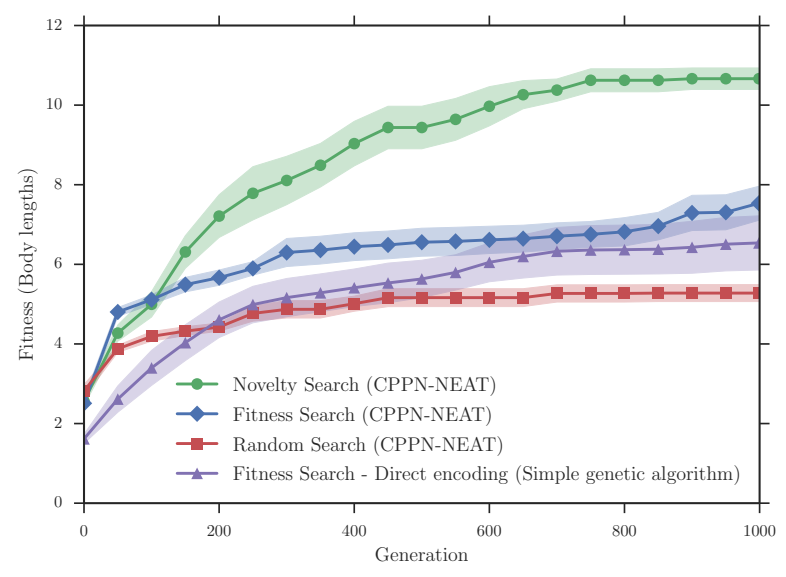

Figure 6: Comparison of simple genetic algorithm (direct encoding) against novelty-fitness-random search with generative encoding. Best fitness so far averaged over 10 runs, deviation error is shown by the shaded part.

Details about the experimental setup, source code, and a wiki page on how to reproduce experiments can be found in the project's repository ${ }^{3}$.

\section{RESULTS}

Pure novelty search is compared with respect to the fitness measure (displacement of soft robots in body-lengths) to fitness-based search. Different behavior types are used to investigate the effects on performance of novelty search. In addition we explore the effect of fitness-elitism when combined with novelty search. Lastly, the performance of both methods, novelty and fitness-based search, are investigated for several levels of gravity. Evolved candidate solutions show how environmental conditions can have significant effect on the morphologies and the locomotion strategies of soft robots.

\subsection{Behavior selection}

Figure 5 illustrates the performance achieved by novelty search for the 10 behavior types. In particular, what is shown is the fitness in body-lengths of the champion of the whole evolution for 10 independent runs for a lattice size $7^{3}$. Both trajectory-type behaviors achieve the best performance with regards to the fitness measure, with a small difference in favor of $2 \mathrm{D}$ over $3 \mathrm{D}$ trajectories. The rest of the behavior metrics apart from VTG and DFT-VTG are close as far as the final performance of the evolution is concerned. One reason they fail to meet the trajectories' performance is the fact that although they keep track of cues that can describe the performance of the robot (e.g., displacement and speed), they cannot encode the direction of motion. Soft robots that have a circular trajectory can exhibit fast locomotion, in this case though, the measured displacement from their initial position remains low. Counting the number of voxels of a soft robot that touched the ground at every sampling step of the simulation does not hold any information as to how fast the robot is moving. A fast moving robot that

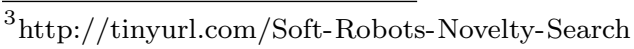




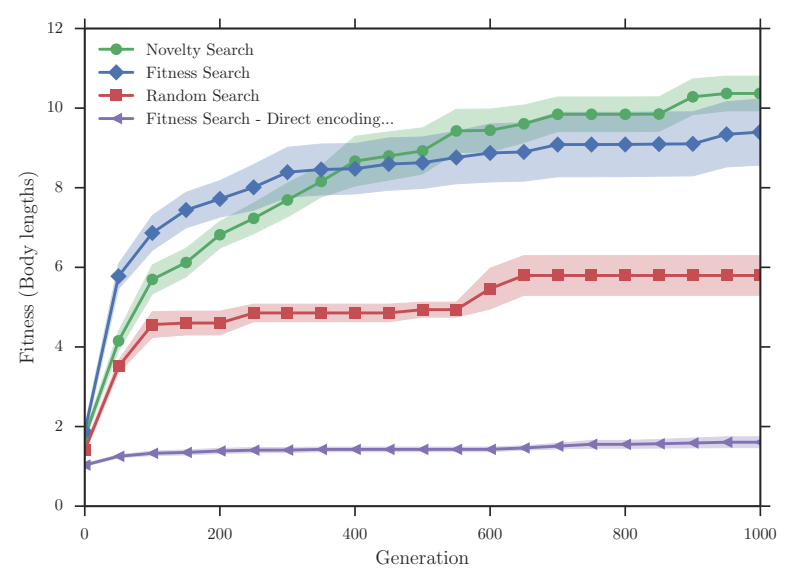

Figure 7: Simple genetic algorithm against noveltyfitness-random search with generative encoding. Best fitness so far averaged over 10 runs.

is hopping can have a similar behavior to a hopping robot that stays in the same position. In contrast, the trajectory behavior of these robots would show a large difference.

\subsection{Performance comparison}

To compare the performance achieved by novelty search, its performance is set side by side with fitness-based search (normalized body-length displacement of the soft robot's center of mass from its initial position), random search, and finally a simple genetic algorithm ${ }^{4}$ with direct encoded genomes. The same experiment held under two different simulation settings (i.e. for lattice size $5^{3}$ and $10^{3}$ ). Note, that the first three methods are referring to a generative encoding evolved by CPPN-NEAT and using selection with respect to novelty, fitness, and random selection. The last method uses a direct encoded genome driven by fitness within a simple genetic algorithm. Two dimensional trajectories are used by novelty search in order to describe the novelty in the behavior space through the sparseness measure.

Figure 6 presents the results for the small lattice (i.e. $5^{3}$ ). The average best displacement so far is presented alongside the deviation error. Note, the difference between novelty search and the other methods. Using the two dimensional trajectories of the soft robots, novelty search visits optimal solutions that none other method reaches. Local optima can prevent fitness-based search to achieve the performance of novelty search. Encoding limitations in direct encoding cannot lead to optimal solutions for this settings. In the case of random search, having neither the information about their fitness, nor the driving force of novelty search that seeks for novel behaviors, it fails to evolve any successful locomotion strategy. The only reason random search in CPPN-NEAT achieves a displacement of $\sim 5$ body-lengths, is the powerful generative encoding. The simple genetic algorithm approach performs better than using random selection with an indirect encoding. Structural regularity and symmetry does not show all of its advantages in this small lattice setting.

For larger lattices, it is expected that generative encoding will prove its merits over the direct encoding scheme [10, 11].

${ }^{4}$ The GAlib $\mathrm{C}++$ library [24] used for the implementation of this method. Source code used from [10].

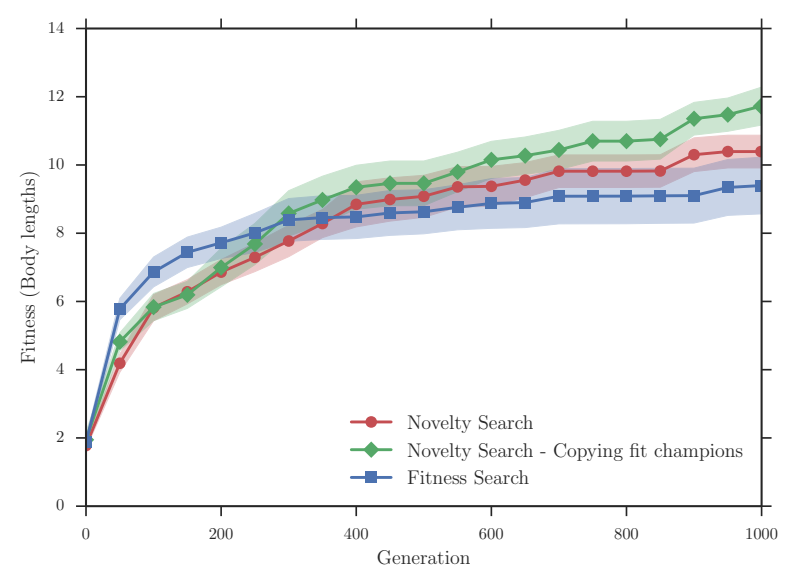

Figure 8: Best fitness so far, novelty search with and without copying fit champions (fitness-elitism), and fitness search, averaged over 10 runs.

More complicated morphologies can be produced (morphology space for $10^{3}$ lattice resolution: $\left.9.3 \times 10^{698}\right)$. Furthermore, the space of behaviors, for instance two dimensional trajectories, becomes larger since more complex soft robots can achieve higher displacement and more advanced strategies for locomotion. These higher resolution morphologies can achieve life-like locomotion. Figure 7 illustrates the performance (i.e. best displacement so far) of the four different methods in these higher resolution settings. Results reassure that novelty search achieves higher fitness ( $>1$ body-length) on average against fitness-based search. Nevertheless, the effect is not as strong as in the previous experiment. Both methods succeed to evolve the soft robot structure with the highest fitness found in all experiments ( $~ 14$ body-lengths). Novelty search behaves more constant in evolving individuals with high fitness in all runs, on the other hand most of individual runs of fitness-based search are being trapped in low fitness local optima, trying to optimize specific individuals without trying to explore deeply the fitness landscape like novelty search successfully does. The high difference between random evolution and novelty search proves that seeking novel behaviors in novelty search cannot be considered as a random search. The superiority of generative encoding (i.e. CPPN) over direct encoding can be clearly observed. Regularity in shape morphologies provides advantages that result in more efficient motion.

\subsection{Fitness-elitism in novelty search}

The reason that novelty search is considered such a revolutionary search method is because it finds solutions for deceptive problems, where the fitness landscape is not a straightforward function. At each generation of novelty search, novel behaviors that are also fit with regards to the objective of the problem are discovered. Mutations of these solutions will yield in behaving similarly to their ancestors, resulting in similar behaviors. Thus, the novelty value of these individuals will be declined as similar behaviors will contribute in a denser area in the behavior space. Eventually, these solutions will stop being selected, and evolution will not have the chance of carrying their genes along. Mutations and other genetic operations can optimize these fit individuals 


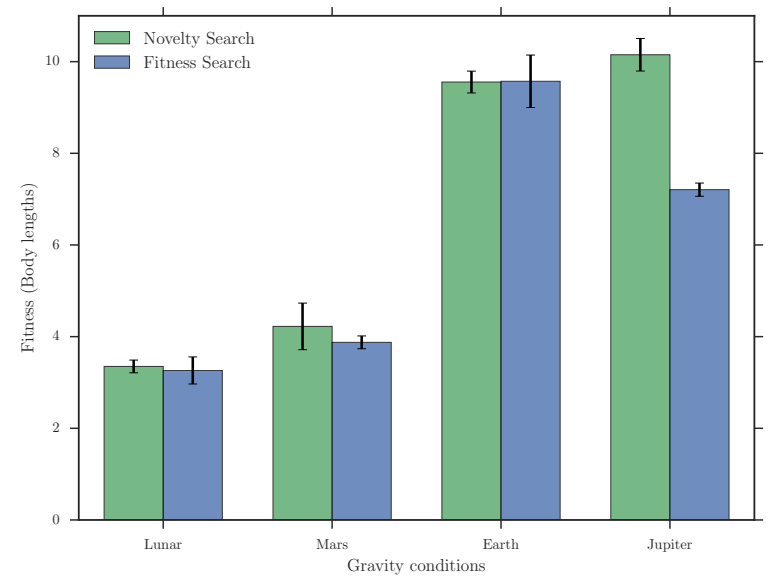

Figure 9: Novelty search performs better or equally good than fitness-based search in all gravity conditions tested.

more. These individuals (with high fitness values) can be seen as stepping stones [16] towards more optimized versions of themselves. Being blind to the objective function, novelty search will eventually stop producing new individuals out of them, which will lead to promising individuals being unable to survive through the evolution process. Figure 8 illustrates the gain in performance when fitness-elitism is used in novelty search method compared with the pure novelty and fitness-based search methods.

\section{EVOLVING SOFT ROBOTS FOR SPACE EXPLORATION}

Gravity conditions heavily affect the evolution of soft robots in our set-up. The interaction between the robot body and the environment biases the selection towards chromosomes that are able to produce robot bodies that efficiently interact with the terrain at the considered gravity strength. We experiment with both methods discussed, novelty and fitness-based search, presenting some interesting resulted body morphologies and locomotion strategies evolved for specific gravity accelerations using a large lattice size $\left(10^{3}\right)$. For the novelty search method we select the two dimensional trajectories of the soft bodies as the behavior metric defining the novelty of each individual. Due to the computational time demand of the simulations especially in lower gravity accelerations (longer simulation trials are needed to observe the locomotion strategy of hopping soft robots), a lower lattice size is used to approximate the performance of both methods under different gravity strengths. Figure 9 presents the performance of novelty and fitness-based search for four different gravity levels using a lattice size $7^{3}$.

\section{Soft robots on Lunar's gravity}

Locomotion strategies evolved under Lunar gravity showed that only hopping gaits can produce effective locomotion. Low gravity makes it challenging for the soft body structures to grip on the ground surface and evolve different strategies than hopping. The morphology of each hopper differs. A Cshaped hopper soft robot (see Fig. 11a) evolved in these set-

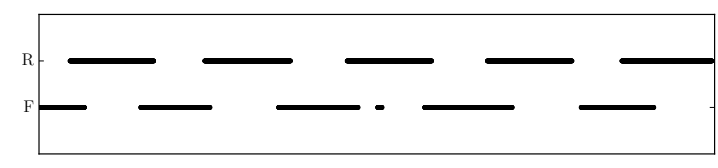

(a) Two-legged soft robot.

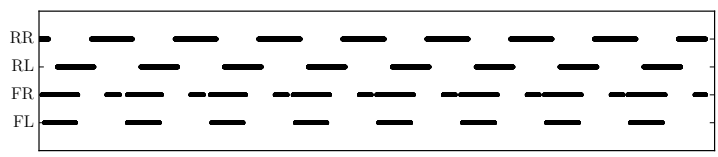

(b) Four-legged soft robot.

Figure 10: Hildebrand diagrams of two evolved soft robots for Earth's gravity acceleration. Timing of impacts between legs and the ground.

tings. Additional experiments showed that lower actuation frequencies for the soft materials contribute in the evolution of stable locomotion strategies, while higher frequencies cannot produce decent motion for the soft bodies.

\section{Soft robots on Mars' gravity}

The locomotion effectiveness on Mars is higher when compared to Lunar, the higher gravity acceleration makes it possible for the virtual soft robots to evolve other kind of gaits using legged bodies (see Fig. 11b). Note that the C-shaped hopper soft robot mostly uses passive materials apart from its upper body where all the active material are located. Its upper part alone generates enough motion to hop efficiently. What is observed in the morphologies evolved at lower gravity levels is that the use of a lower number of active voxels can still produce decent locomotion. Apart from hopping strategies, other motion behaviors were observed including legged soft robots.

\section{Soft robots on Earth's gravity}

On higher gravity levels familiar locomotion strategies emerge. In Figure 10 we show the Hildebrand diagram for two of the creatures evolved under Earth gravity, where we note characteristics remarkably similar to biped and quadruped locomotion used by animals on Earth. Interesting animallike gaits are visualized in Fig. 11c. These results suggest an encouraging connection between our set-up and the locomotion strategies of living organisms evolved on Earth for thousands of years. Tumbleweed-like locomotion (see Fig. 11d) has also emerged at this gravity level under novelty search, producing rolling soft robots that can locomote efficiently. Tumbleweed is also a concept of low-cost exploration that has inspired robot designers for Mars' missions in the past [2] and has been already deployed in Antarctica for testing purposes by NASA.

\section{Soft robots on Jupiter's gravity}

Moving on to higher gravity levels, i.e. on Jupiter (assuming a stable surface), heavier structures can use galloping as a strategy for their locomotion. Galloping is again consid- 

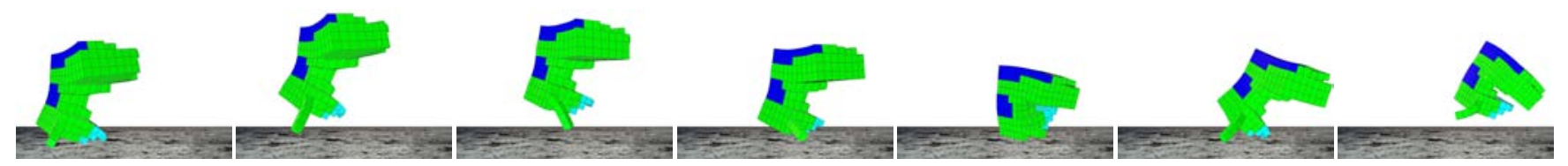

(a) Lunar's gravity: C-shaped hopper (novelty search)

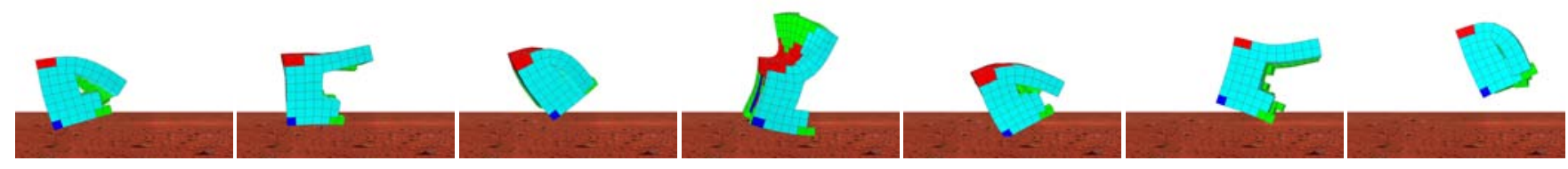

(b) Mars' gravity: 2-legged C-shaped hopper (novelty search)
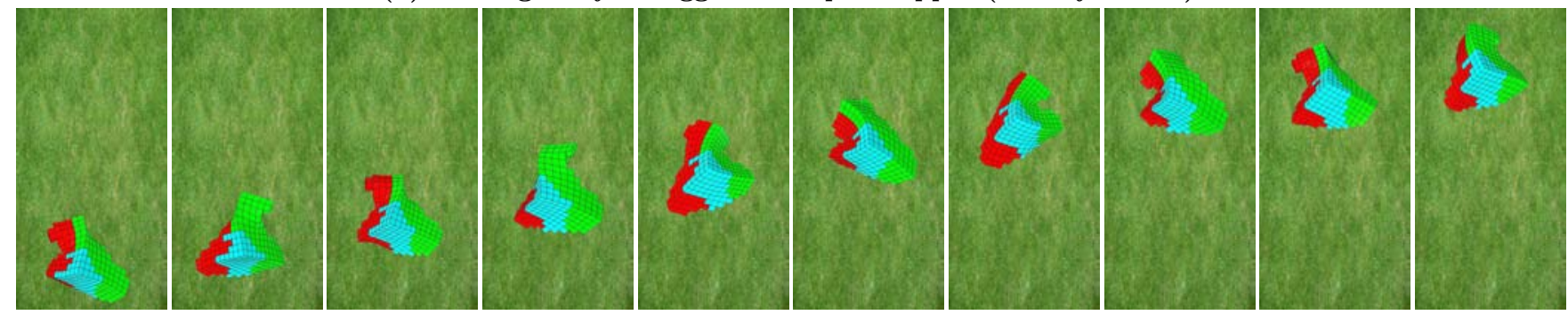

(c) Earth's gravity: Top view, 4-legged animal like locomotion (fitness search)

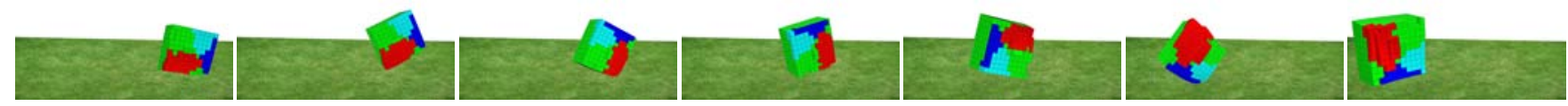

(d) Earth's gravity: Tumbleweed-like locomotion (novelty search)

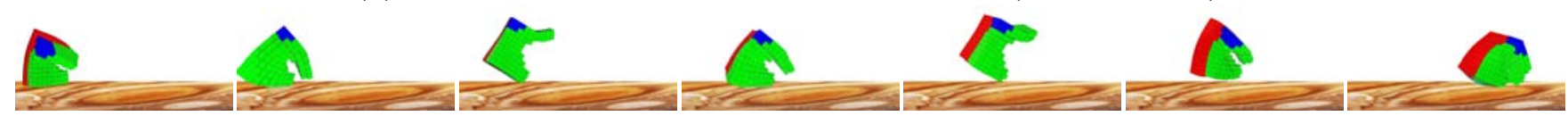

(e) Jupiter's gravity (Assuming a stable surface): C-shaped hopper (novelty search)

Figure 11: Locomotion strategies evolved in variant gravity conditions.

ered to be an effective way of moving in such a high gravity, whereas thicker legs are evolved to withstand the high gravitational force. Push-pull worm-like locomotion can also produce decent velocities for soft robots. Finally, hoppers have also evolved in this setting (performing short hops), while using more actuated materials (see Fig. 11e) than the hopping soft robots presented earlier for lower gravity accelerations.

\section{DISCUSSION AND OUTLOOK}

In all our experiments, both novelty search and fitness search evolved soft bodies able to efficiently move in the considered environment. However, the performance with regard to the fitness defined (i.e. displacement of the body in bodylengths) was equal or higher for novelty search in all experiments. One would expect novelty to deliver more diversity, at the cost of a lower fitness function; novelty does not know about fitness and a method well targeted to improve fitness is likely to deliver better results. This was not the case and we found that the creatures evolved with novelty are also able to move faster than the ones that were evolved to move fast. Previous work [19] in a try to evolve walking three dimensional virtual creatures used the evolved morphology of the creatures to describe their behavior. Although, comparing the morphology of the evolved soft robots is similar to comparing the chromosome (CPPN) of each individual. Be- haviors that describe the morphology of the evolved robots have failed [19], since search is then forcing new types of morphologies without caring about the actual target of the evolution, which was the efficient locomotion. Therefore, only the comparison of the observed behavior in the phenotype level can lead the evolution towards more complex behaviors.

Some interesting general observations can be made. To start with, low gravity levels seem not to allow for a great variety of gaits. Hopping seems to be the predominant strategy followed by most evolved creatures. Higher gravity levels, instead, allow for more complicated behaviors to emerge and legged locomotion starts to became favorable. At Earth gravity levels, gaits remarkably similar to animal-like motion appear, increasing our confidence in the simulation setup. Second, the greater variety of creatures evolved using novelty search, allow for rolling tumbleweed-like [2] robots, quadruped and biped robots, hoppers as well as odd creatures to emerge.

Beyond its purely inspirational value, we believe that this methodology, if improved and refined, can be of direct use for the design of soft robotic rovers. Extreme temperature fluctuations, for example on asteroids or comets, could trigger passive actuated materials to perform a designed movement. Although the VoxCad simulator could not be used to simulate the extreme environment of asteroids or comets, it is an interesting idea for further research. The rotation 
of the celestial body (e.g., with a period as short as a few hours) would then create a cyclic temperature profile having an extreme excursion and thus actuating the soft robot locomotion. This would result, if the soft robot was evolved in such an extreme environment, into a slow crawl of the robot leading to an extremely useful exploratory behavior that could deliver incredible scientific data.

\section{CONCLUSIONS}

We introduced the use of a diversity seeking method, novelty search, in evolutionary soft robotics. We found that well defined behavior metrics can lead novelty search to outperform traditional fitness-based search. Novelty search not only improved the performance and the diversity in the fitness space, but also contributed to a larger variety of morphologies. Finally, both techniques were used to evolve soft robots in four different gravity levels, showing interesting results and the possibility of influencing future robotic designs for planetary exploration.

\section{REFERENCES}

[1] Georgios Methenitis. Evolution of Soft Robots by Novelty Search. Master's thesis, University of Amsterdam, The Netherlands, 2014.

[2] Jeffrey Antol, Philip Calhoun, John Flick, Gregory Hajos, Richard Kolacinski, David Minton, Rachel Owens, and Jennifer Parker. Low cost mars surface exploration: the mars tumbleweed. Technical report, 2003.

[3] Tomi Ylikorpi, Aarne Halme, Peter Jakubik, Jussi Suomela, and Mika Vainio. Biologically inspired solutions for robotic surface mobility. In Proceedings of 8th ESA Workshop on Advanced Space Technologies for Robotics and Automation, pages 2-4, 2004.

[4] PE Clark, SA Curtis, ML Rilee, and SR Floyd. Ali (autonomous lunar investigator): Revolutionary approach to exploring the moon with addressable reconfigurable technology. In 36th Annual Lunar and Planetary Science Conference, volume 36, page 1217, 2005.

[5] Evangelos G Papadopoulos, Ioannis Kontolatis, Iosif S Paraskevas, and Leopold Summerer. Ariadna study: Space gaits. Technical report, 2013.

[6] Ioannis Kontolatis, Dimitrios Myrisiotis, Iosif Paraskevas, Evangelos Papadopoulos, Guido de Croon, and Dario Izzo. Quadruped optimum gaits analysis for planetary exploration. In 12th Symposium on Advanced Space Technologies in Robotics and Automation.

[7] M. Hildebrand. Vertebrate locomotion: An introduction: How does an animal's body move itself along? BioScience, 39(11):764-765, 1989.

[8] Karl Sims. Evolving virtual creatures. In Proceedings of the 21st annual conference on Computer graphics and interactive techniques, pages 15-22. ACM, 1994.

[9] Jonathan Hiller and Hod Lipson. Automatic design and manufacture of soft robots. Robotics, IEEE Transactions on, 28(2):457-466, 2012.

[10] Nick Cheney, Robert MacCurdy, Jeff Clune, and Hod Lipson. Unshackling evolution: evolving soft robots with multiple materials and a powerful generative encoding. In Proceeding of the fifteenth annual conference on Genetic and evolutionary computation conference, pages 167-174. ACM, 2013.

[11] Kenneth O Stanley. Compositional pattern producing networks: A novel abstraction of development. Genetic programming and evolvable machines, 8(2):131-162, 2007.

[12] Deepak Trivedi, Christopher D Rahn, William M Kier, and Ian D Walker. Soft robotics: Biological inspiration, state of the art, and future research. Applied Bionics and Biomechanics, 5(3):99-117, 2008.

[13] Rolf Pfeifer, Max Lungarella, and Fumiya Iida. The challenges ahead for bio-inspired'soft'robotics. Communications of the ACM, 55(11):76-87, 2012.

[14] H. Lin, G.G. Leisk, and B.A. Trimmer. Soft robots in space: A perspective for soft robotics. Acta Futura, 6:69-79, 2013.

[15] Joel Lehman and Kenneth O Stanley. Exploiting open-endedness to solve problems through the search for novelty. In ALIFE, pages 329-336, 2008.

[16] Joel Lehman and Kenneth O Stanley. Abandoning objectives: Evolution through the search for novelty alone. Evolutionary computation, 19(2):189-223, 2011.

[17] Joel Lehman and Kenneth O Stanley. Revising the evolutionary computation abstraction: minimal criteria novelty search. In Proceedings of the 12th annual conference on Genetic and evolutionary computation, pages 103-110. ACM, 2010.

[18] Sebastian Risi, Sandy D Vanderbleek, Charles E Hughes, and Kenneth O Stanley. How novelty search escapes the deceptive trap of learning to learn. In Proceedings of the 11th Annual conference on Genetic and evolutionary computation, pages 153-160. ACM, 2009.

[19] Joel Lehman and Kenneth O Stanley. Evolving a diversity of virtual creatures through novelty search and local competition. In Proceedings of the 13th annual conference on Genetic and evolutionary computation, pages 211-218. ACM, 2011.

[20] Michael T Tolley, Robert F Shepherd, Bobak Mosadegh, Kevin C Galloway, Michael Wehner, Michael Karpelson, Robert J Wood, and George M Whitesides. A resilient, untethered soft robot. Soft Robotics, 1(3):213-223, 2014.

[21] Jonathan Hiller and Hod Lipson. Dynamic simulation of soft heterogeneous objects. arXiv preprint arXiv:1212.2845, 2012.

[22] Kenneth Stanley and Risto Miikkulainen. Evolving neural networks through augmenting topologies. Evolutionary computation, 10(2):99-127, 2002.

[23] Jean-Baptiste Mouret. Novelty-based multiobjectivization. In New Horizons in Evolutionary Robotics, pages 139-154. Springer, 2011.

[24] Matthew Wall. Galib: A c++ library of genetic algorithm components. Mechanical Engineering Department, Massachusetts Institute of Technology, 87:54, 1996. 